\title{
Estimativa da área foliar do meloeiro irrigado em função do número de folhas
}

Dados de área foliar são comumente utilizados em diversos experimentos na agricultura, no entanto, geralmente, são determinados utilizando-se de equipamento caros e análises destrutivas. Diversos métodos têm sido desenvolvidos para estimar a área foliar de folhas que comumente empregam medidas como comprimento e largura, entretanto, são métodos trabalhosos pelo fato de exigir que a medição seja feita de forma individual. Assim, o presente trabalho faz uso de uma relação de regressão para estimar de forma não destrutiva a área foliar do meloeiro, envolvendo apenas o número de folhas. 0 método foi testado em 5 cultivares de melão: Melody (melão amarelo), Goldex (melão amarelo), pele-de-sapo, AF-646 (melão amarelo), Orange Flesh e Torreon (cantaloupe). Os valores de área folia e número de folhas foram obtidos em pelo menos cinco épocas distintas durante o ciclo da cultura, totalizando 938 amostras. As equações ajustadas para cada cultivar foram comparadas pelo teste de identidade de modelo para se verificar a hipótese de nulidade de que as equações são iguais estatisticamente. Para as 5 cultivares, os coeficientes de determinação (R2) entre a área foliar medida real e a área foliar estimada foram iguais ou maiores a 0,9. Assim, a relação encontrada apresenta-se como uma ferramenta promissora para pesquisas que exigem estimativas não destrutivas precisas da área foliar de meloeiros.

Palavras-chave: Modelagem; Análise de Crescimento; Área Foliar.

\section{Estimate of the leaf area in function of leaf number in muskmelon}

Leaf area data are commonly used in various experiments in agriculture, however, they are usually determined using expensive equipment and destructive analysis Several methods have been developed to estimate the leaf area of leaves that commonly employ measures such as length and width, however, they are laborious methods because they require that the measurement be made individually. Thus, the present work uses a regression relation to estimate non-destructively the melon leaf area, involving only the number of leaves. The method was tested on 5 melon cultivars: Melody (yellow melon), Goldex (yellow melon), Santa Claus melon, AF-646 (yellow melon), Orange Flesh and Torreon (cantaloupe). The values of leaf area and number of leaves were obtained at least five different times during the culture cycle, totaling 938 samples. The adjusted equations for each cultivar were compared using the model identity test to verify the null hypothesis that the equations are statistically equal. For the 5 cultivars, the determination coefficients (R2) between the actual measured leaf area and the estimated leaf area were equal to or greater than 0.9 . Thus, the relationship found presents itself as a promising tool for research that requires precise non-destructive estimates of melon leaf area.

Keywords: Modeling; Growth Analysis; Leaf Area.

Topic: Botânica Agrícola

Reviewed anonymously in the process of blind peer.
Received: 09/02/2020

Approved: 09/03/2020
Celsemy Eleutério Maia (ic

Universidade Federal Rural do Semi-Árido, Brasil

http://lattes.cnpq.br/1217682039861125

http://orcid.org/0000-0002-2253-3022

celsemy@ufersa.edu.br

José Mariano da Silva Neto

Universidade Federal de Campina Grande, Brasil

http://lattes.cnpq.br/1035801819440482

http://orcid.org/0000-0002-2427-1855

neto-silva@hotmail.com

Ana Quézia Carvalho Braga (iD

Universidade Federal Rural do Semi-Árido, Brasil

http://lattes.cnpq.br/9196532878983547

http://orcid.org/0000-0002-9859-1720

queziaana@outlook.com
Referencing this:

MAIA, C. E.; SILVA NETO, J. M.; BRAGA, A. Q. C.. Estimativa da área foliar do meloeiro irrigado em função do número de folhas. Revista Ibero Americana de Ciências Ambientais, v.11, n.2, p.85-91, 2020. DOI: http://doi.org/10.6008/CBPC2179-6858.2020.002.0010 


\section{INTRODUÇÃO}

O melão (Cucumis melo L.), é uma hortaliça tipo fruto, pertencente à família Curcubitaceae, é uma das frutas mais populares e cultivadas no mundo (WU et al., 2020). O Brasil produziu, em 2018, 581.478 toneladas de melão com uma área de colheita de 23.324 hectares. A determinação de área foliar (AF) no cultivo de frutas é um processo importante, porém difícil de estabelecer uma precisão necessária para a obtenção de dados confiáveis, devido ao grande número de folhas e à complexidade da estrutura tridimensional dos dóceis das árvores (SANZ et al., 2018).

Essa determinação é de fundamental importância pelo fato das folhas estarem relacionadas a diversos processos com à evapotranspiração, interceptação de radiação e fixação de $\mathrm{CO}_{2}$ (HERNANDEZSANTANA et al., 2017), bem como, para a obtenção de alguns parâmetros fisiológicos, tais como taxa de assimilação líquida, razão de área foliar, área foliar específica, índice de área foliar e razão de peso de folha, muito utilizados na análise de crescimento de plantas (CAIRO et al., 2008).

Numerosos estudos têm sido desenvolvidos para a obtenção de métodos que utilizem parâmetros mais fáceis de se obter, com baixo custo em termos de mão de obra e tempo, que os tornem viáveis para fins comerciais. Existem dois tipos principais de métodos: diretos ou destrutivos, que se caracterizam por medir diretamente a área da superfície foliar e os indiretos ou não-destrutivos que usam outros parâmetros que estão relacionados a área foliar e que que são mais fáceis de se obter (ROSSEL et al., 2012).

Uma vez que área foliar é uma característica difícil de ser mensurada, requerendo equipamentos caros ou técnicas destrutivas, torna-se muito importante à determinação de uma equação que possa estimar a área foliar das plantas a partir de valores, que podem ser obtidos de forma não-destrutiva (LIMA et al., 2017). De acordo com Spann et al. (2010) métodos não destrutivos precisos e simples para estimar a área da folha são essenciais para muitos estudos fisiológicos, ecológicos, hortícolas e agronômicos.

Os métodos não destrutivos já foram reportados na literatura para uma grande variedade de culturas como soja (SOUZA et al., 2019), acerola (AZEVEDO et al., 2019), cacau (SCHMILDT et al., 2017) e morango (ZEIST et al., 2014). Nesses métodos as medidas são tomadas na planta, sem necessidade de remoção de estruturas, preservando sua integridade e permitindo a continuidade das medidas na mesma planta.

Frequentemente, os estudos dos fenômenos ecofisiológicos requerem um método não destrutivo de medida da área foliar. Em tais casos, uma alternativa para a obtenção da medida direta da área foliar é o desenvolvimento de fórmulas ou equações matemáticas através das quais se possa estimar tal área, como uma função das dimensões lineares, como o comprimento e a largura, podendo-se utilizar também a estimativa da área foliar por modelos matemáticos, sendo que a variável independente deve ser obtida da forma mais simples e rápida, assim, o objetivo desse trabalho foi estimar a área foliar de cinco cultivares de meloeiro irrigado em função do número de folhas.

\section{METODOLOGIA}

Para o estudo da área foliar em função do número de folhas, utilizou-se de um banco de dados 
composto por valores de área foliar e seu respectivo número de folhas por planta das seguintes cultivares de melão: Melody (melão amarelo), Goldex (melão amarelo), pele-de-sapo, AF-646 (melão amarelo), Orange Flesh e Torreon (cantaloupe). Esses dados com diferentes tratamentos foram realizados na região de Mossoró no estado do Rio Grande do Norte. Os valores de área foliar e número de folhas foram obtidos em pelo menos cinco épocas distintas durante o ciclo da cultura, totalizando 938 amostras, sendo a quantidade por cultivar de acordo com a Tabela 1.

Tabela 1: Número de amostras por cultivar.

\begin{tabular}{|l|l|l|}
\hline \multicolumn{2}{|l|}{ Cultivar de Melão } & Número de amostras \\
\hline $\mathbf{M}_{\mathbf{1}}$ & Orange Flesh & 50 \\
\hline $\mathbf{M}_{\mathbf{2}}$ & Melody & 72 \\
\hline $\mathbf{M}_{\mathbf{3}}$ & Goldex & 341 \\
\hline $\mathbf{M}_{\mathbf{4}}$ & Pele de Sapo & 44 \\
\hline $\mathbf{M}_{\mathbf{5}}$ & AF-646 & 390 \\
\hline $\mathbf{M}_{\mathbf{6}}$ & Torreon & 41 \\
\hline Total & 938 \\
\hline
\end{tabular}

O número de folhas foi obtido pela contagem das mesmas totalmente expandidas, consideradas no mínimo, 10 folhas por planta. Sendo retirada uma planta de cada parcela experimental em cada experimento. A área foliar foi obtida através de um integrador de área, marca LI-COR, modelo LI-3100.

Para análise estatística, foram realizados ajustes do modelo linear tendo como variável dependente a área foliar e independente o número de folhas. As equações ajustadas para cada cultivar foram comparadas pelo teste de identidade de modelo para se verificar a hipótese de nulidade de que as equações são iguais estatisticamente. $\mathrm{O}$ teste de identidade de modelo baseia-se na diferença entre a soma dos quadrados de parâmetros do modelo completo e a soma de quadrados de parâmetros do modelo reduzido (REGAZZI et al., 1996). Inicialmente, fez-se o teste para todas as equações ajustadas para cada cultivar de melão (Tabela 1); depois, o teste de identidade de modelo, comparando-se cada equação duas a duas. As análises foram realizadas utilizando o software SAEG, desenvolvido pela Universidade Federal de Viçosa, MG.

A área foliar foi avaliada pelo valor estimado em função do observado e se utilizou a metodologia proposta por Gauche et al. (2003), que leva em consideração a decomposição do quadrado médio dos desvios (MSD) em três componentes: quadrado da bias (SB), declividade não unitária (NU) e falta de correlação (LC), que corresponde a:

$$
\begin{aligned}
& M S D=\frac{\sum\left(X_{n}-Y_{n}\right)^{2}}{N} \\
& S B=(\bar{X}-\bar{Y})^{2} \\
& N U=(1-b)^{2} \cdot\left(\frac{\sum\left(X_{n}-\bar{X}\right)^{2}}{N}\right) \\
& L C=\left(1-r^{2}\right) \cdot\left(\frac{\sum\left(Y_{n}-\bar{Y}\right)^{2}}{N}\right) \\
& M S D=S B+N U+L C
\end{aligned}
$$


Onde:

$X_{n}, \bar{X}=$ valores observados e médios, respectivamente; $Y_{n}, \bar{Y}=$ valores estimados e médios, respectivamente;

$N$ = número de pares de dados usados no modelo; $b=$ declividade da reta dos valores estimados em função dos observados; $r=$ coeficiente de correlação entre os valores estimados e observados.

\section{RESULTADOS E DISCUSSÃO}

Na Figura 1, observa-se a relação entre a área foliar (AF) e o número de folhas (NF), das cultivares de meloeiro estudadas, independentemente do tempo de plantio. O número de folhas variou de 10 a 222 folhas e a área foliar de 301,75 a $26.462,73 \mathrm{~cm}^{2} /$ planta, sendo observado o comportamento linear entre as duas variáveis, em que a área foliar pode ser estimada em função do número de folhas pela equação 6 , com coeficiente de determinação de 0,8860 .

$$
A F=-321,82+99,54 \times N F
$$

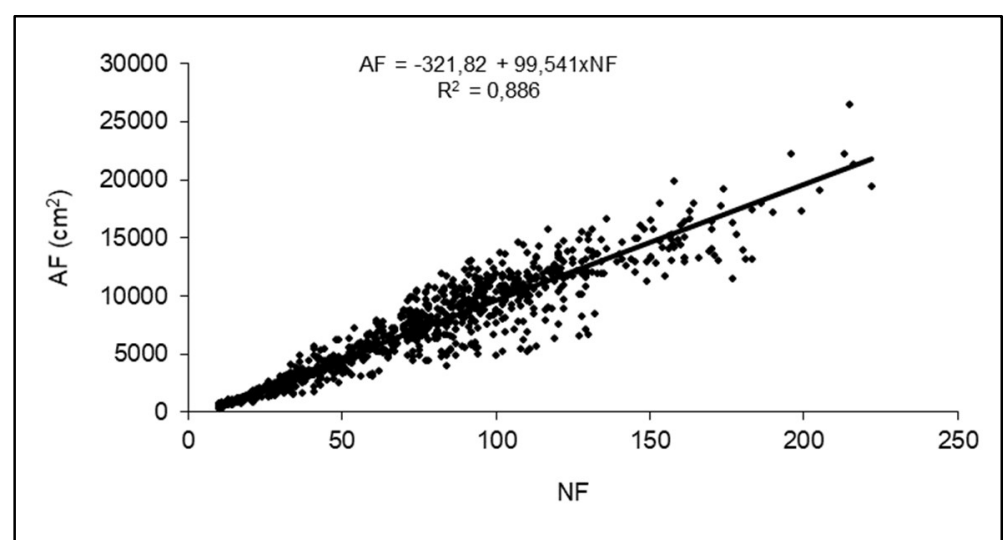

Figura 1: Relação da área foliar do meloeiro em função do número de folhas na planta, independente da época e da cultivar.

O efeito linear da relação área foliar x número de folhas também foi observado por Nascimento et al. (2002) estimando a área foliar do meloeiro em função do número de folhas e das suas dimensões, observaram que a área foliar pode ser determinada com precisão razoável a partir do número de folhas, independente da salinidade da água e da lâmina de irrigação aplicada e idade da planta. Queiroga et al. (2008) também verificaram efeito linear entre área foliar e número de folhas.

Tabela 2: Parâmetros das equações de regressão linear $(Y=a+b X)$, coeficientes de determinação e teste de identidade de modelos por melão.

\begin{tabular}{|c|c|c|c|}
\hline \multirow[t]{2}{*}{ Cultivar de Melão } & \multicolumn{3}{|c|}{$A F=a+b x N F$} \\
\hline & $a$ & $\mathrm{~b}$ & $\mathrm{R}^{2}$ \\
\hline $\mathrm{M}_{1}$ & 267,05 & $56,29^{* *}$ & 0,9151 \\
\hline$M_{2}$ & $-350,66$ & $106,86^{* *}$ & 0,9647 \\
\hline$M_{3}$ & $-671,47$ & $114,34^{* *}$ & 0,9210 \\
\hline $\mathrm{M}_{4}$ & $-71,27$ & $60,86^{* *}$ & 0,9720 \\
\hline$M_{5}$ & $-411,56$ & $99,13^{* *}$ & 0,9190 \\
\hline$M_{6}$ & $-332,24$ & $93,41^{* *}$ & 0,9489 \\
\hline Conjunta $\mathrm{M}_{1}$ e $\mathrm{M}_{4}$ & 53,25 & $58,50^{* *}$ & 0,9542 \\
\hline Conjunta $\mathrm{M}_{2}$ e $\mathrm{M}_{3}$ & $-616,53$ & $113,27^{* *}$ & 0,9286 \\
\hline Conjunta $\mathrm{M}_{5}$ e $\mathrm{M}_{6}$ & $-419,86$ & $98,91^{* *}$ & 0,9226 \\
\hline
\end{tabular}

\footnotetext{
** significativo a $1 \%$ de probabilidade
} 
Na Tabela 2 encontram-se os resultados das análises de regressão com as seis cultivares de melão separadamente, em que as equações são utilizadas para encontrar a área foliar para cada cultivar em função do número de folhas de forma indireta e com um grau de precisão mais elevado do que a equação geral para todas as cultivares. Todas as equações apresentadas permitem obter estimativas satisfatórias da área foliar, onde se observa que o menor coeficiente de determinação paras as cinco cultivares foi de 0,9151.

Nesse contexto, o uso da equação linear apresenta maior facilidade de cálculo para estimar a área foliar, principalmente em relação ao modelo potencial observado na literatura, como o de Lima et al. (2007), que estudando feijão caupi, encontraram equações potenciais com alto coeficiente de determinação, porém as equações lineares envolvendo o comprimento e a largura. Bianco et al. (2008) estudando esta relação em Typha latifolia, obtiveram equações que apresentavam caráter geométrico e exponencial, sendo ainda mais difícil a realização do cálculo, necessitando de meios eletrônicos para encontrar o valor da área foliar, além de possuir baixo coeficiente de determinação, com valores menores que 0,60.

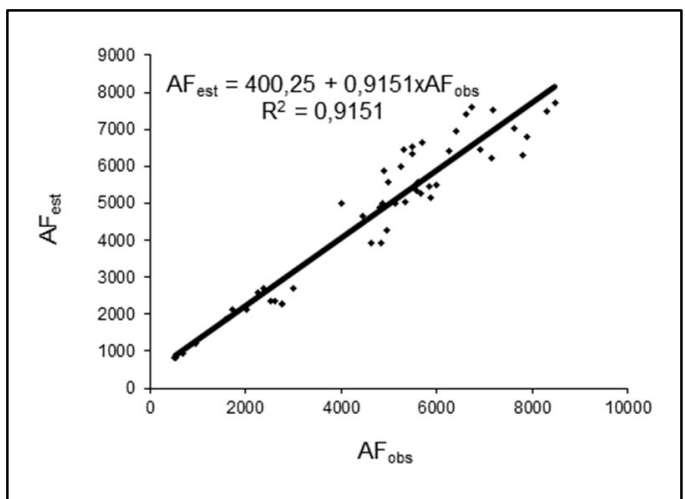

(a) Orange Flesh

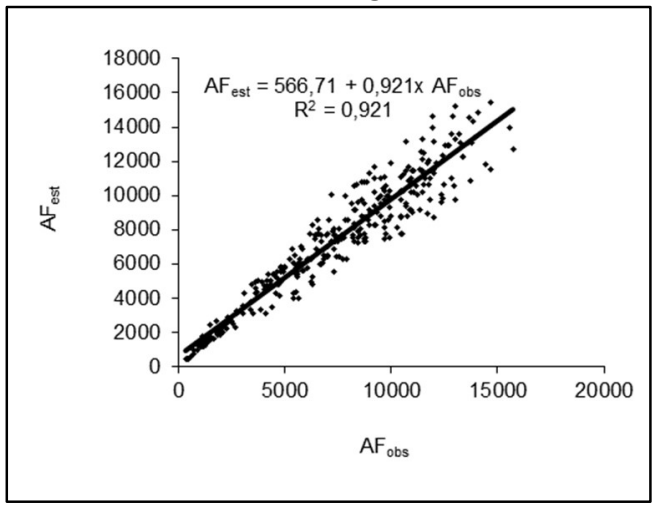

(c) Goldex

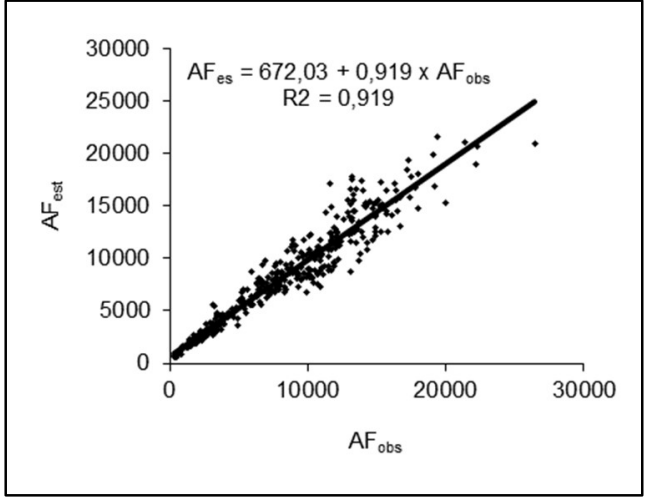

(e)

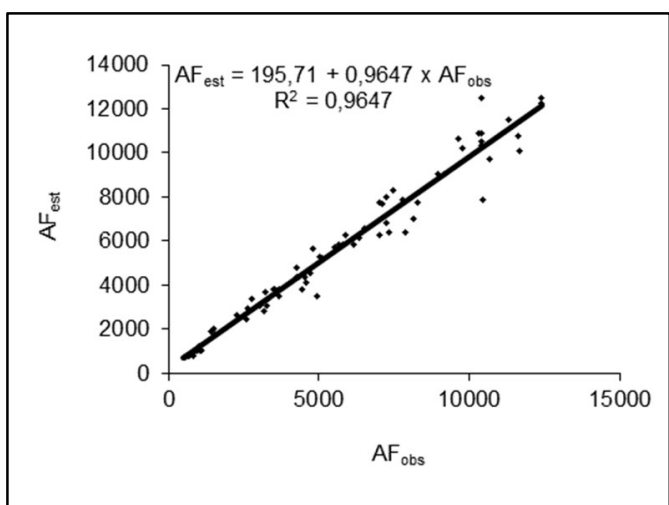

(b) Melody

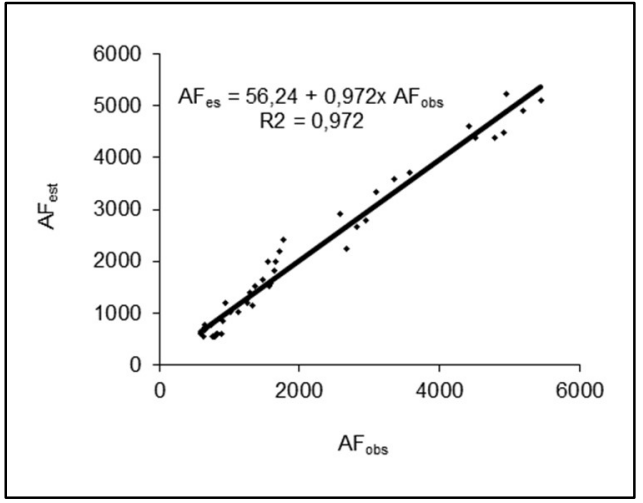

(d) Pele-de-sapo

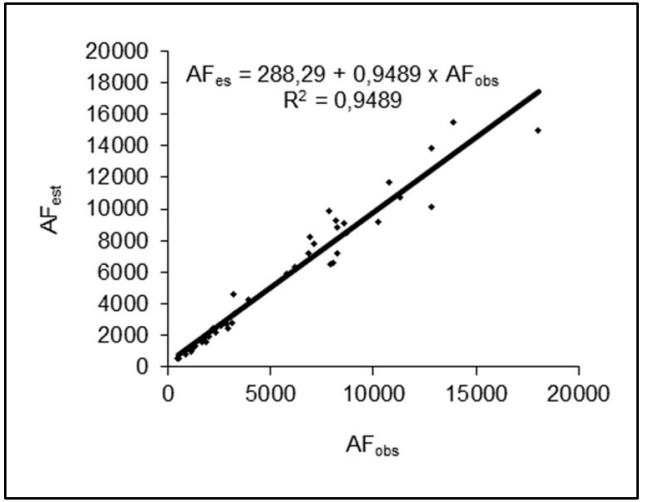

(f) Torreon

Figura 2: Relação da área foliar do meloeiro em função do número de folhas na planta e a relação entre a área foliar observada e a área foliar estimada para as cultivares (a) Orange Flesh, (b) Melody, (c) Goldex, (d) Pele de sapo, (e) AF646 e (f) Torreon. 
Na comparação das equações de regressão através da análise de identidade de modelo, foi feito primeiro para todas as regressões e, depois, comparando-se as equações duas a duas (Tabela 2), dando um total de 15 combinações. Ao se comparar todas as equações ajustadas constatou-se que as cultivares apresentaram diferença estatística entre os modelos ajustados e, quando comparadas as cultivares, duas a duas, $20 \%$ das comparações foram estatisticamente significativas.

Analisando a relação área foliar observada $\left(\mathrm{AF}_{\mathrm{Obs}}\right)$ e estimada $\left(\mathrm{AF}_{\mathrm{Est}}\right)$, como indicado nas Figuras $2 \mathrm{a}$, $2 b, 2 c, 2 d, 2 e$ e $2 f$, pode-se verificar que os coeficientes angulares das equações da reta apresentam valores próximos à unidade, de forma que todas as equações possibilitam estimar a área foliar com satisfatória precisão.

As equações de regressão para as cultivares foram analisadas duas a duas, pelo teste de identidade de modelo e verificou-se que, as cultivares $M_{1}$ e $M_{4} ; M_{2}$ e $M_{3}$; e $M_{5}$ e $M_{6}$, são iguais estatisticamente, ou seja, que para cada par de cultivares pode-se utilizar a mesma equação de regressão para se estimar a área foliar em função do número de folhas, como especificado na Tabela 2. Para as cultivares $M_{1}$ e $M_{4}$, pode-se utilizar a equação 7 para a determinação da área foliar, ao invés de cada equação especificamente, apresentando coeficiente de determinação de 0,9542. As cultivares $M_{2}$ e $M_{3}$, também apresentaram igualdade estatística, podendo-se utilizar a equação 8, com o coeficiente de determinação de 0,9286 e, para as cultivares $M_{5}$ e $M_{6}$, verificou-se que as equações para as duas cultivares podem ser substituídas pela equação 9, com coeficiente de determinação 0,9226.

$$
\begin{aligned}
& A F=53,25+58,50 \times N F \\
& A F=-616,53+113,27 \times N F \\
& A F=-419,86+98,91 \times N F
\end{aligned}
$$

Conforme mostra a Tabela 3, avaliando as cultivares pelos valores estimados em função dos observados com relação ao quadrado médio dos desvios (MSD), observa-se que estes valores foram relativamente baixos. Segundo Gauche et al. (2003) quanto menor o valor de MSD, melhor o modelo e menor o desvio dos dados observados. Na decomposição do MSD, observa-se que a falta de correlação (LC) contribui mais para o MSD, enquanto que o quadrado da bias (SB) não teve influência sobre o erro, porém, mesmo o LC sendo maior na decomposição do MSD, os valores dos coeficientes de correlação foram todos superiores a 0,90, indicando que, mesmo o LC sendo maior, este não implica em falta de ajuste do modelo, levando-se em consideração que o MSD foi baixo. Assim, pode-se afirmar que todas as equações apresentaram bom ajuste aos dados.

Tabela 3: Valores de quadrado de bias (SB), declividade não unitária (NU) e falta de correlação (LC), quadrado médio dos desvios (MSD) e coeficiente de correlação $(r)$ dos valores estimados em função dos observados

\begin{tabular}{|l|l|l|l|l|l|l|}
\hline & $\mathrm{M}_{1}$ & $\mathrm{M}_{2}$ & $\mathrm{M}_{3}$ & $\mathrm{M}_{4}$ & $\mathrm{M}_{5}$ & $\mathrm{M}_{6}$ \\
\hline $\mathrm{SB}$ & 0,0000 & 0,0005 & 0,0000 & 0,0000 & 0,0000 & 0,0000 \\
\hline $\mathrm{NU}$ & 328,73 & 148,09 & 917,44 & 17,05 & 1582,84 & 482,82 \\
\hline LC & 3544,74 & 4044,33 & 10700,39 & 592,37 & 17949,74 & 8963,04 \\
\hline MSD & 3873,47 & 4192,41 & 11617,83 & 609,42 & 19532,58 & 9445,86 \\
\hline $\mathrm{r}$ & 0,9151 & 0,9647 & 0,9210 & 0,9720 & 0,9190 & 0,9489 \\
\hline
\end{tabular}




\section{CONCLUSÕES}

De acordo com os resultados obtidos conclui-se que a área foliar do meloeiro irrigado pode ser estimada a partir do número de folhas e, para as cultivares Orange Flash e Pele-de-Sapo a área foliar pode ser estimada por uma única equação linear, assim como para a cultivar Melody e Goldex, como também para AF-646 e Torreon.

\section{REFERÊNCIAS}

AZEVEDO, A. M.; SILVEIRA, V. A.; OLIVEIRA, C. M.; PEDROSA, C. E.; LEMOS, V. T.; VALADARES, N. R.; GUIMARÃES, A. G.. Predição da área foliar em acerola por redes neurais e regressão múltipla. Revista Agrária Acadêmica, Imperatriz, v.2, n.3, p.96-105, 2019. DOI: http://doi.org/10.32406/v2n32019/96-105/agrariacad

BIANCO, S.; BIANCO, M. S.; CARVALHO, L. B.. Estimativa da área foliar de Ageratum conyzoides geratum usando dimensões lineares do limbo foliar. Acta Scientia Agronômica, Maringá, v.30, n.4, p.519-523, 2008. DOI: http://dx.doi.org/10.4025/actasciagron.v30i4.5311

CAIRO, P. A. R.; OLIVEIRA, L. E. M.; MESQUITA, A. C.. Análise de crescimento de plantas. Vitória da Conquista: UESB, 2008.

GAUCHE, G. H.; HWANG, J. T. G.; FICK, G. W.. Model evaluation by comparison of model-based predictions and measured values. Agronomy Journal, Madison, v.95, p.14421446, 2003. DOI:

http://dx.doi.org/10.2134/agronj2003.1442

HERNANDEZ-SANTANA, V.; FERNANDEZ, J. E.; CUEVAS, M. V.; PEREZ-MARTIN, A.; DIAZ-ESPEJO, A.. Photosynthetic limitations by water deficit: Effect on fruit and olive oil yield, leaf area and trunk diameter and its potential use to control vegetative growth of super-high density olive orchards. Agricultural Water Management, v.184, p.9-18, 2017. DOI: http://doi.org/10.1016/j.agwat.2016.12.016

LIMA, C. J. G. S.; OLIVEIRA, F. A.; MEDEIROS, J. F.; OLIVEIRA, M. K. T.; OLIVEIRA FILHO, A. F.. Modelos matemáticos para estimativa de área foliar de feijão caupi. Revista Caatinga, Mossoró, v.21, n.1, p.120-127, 2007.

NASCIMENTO, I. B.; FARIAS, C. H. A.; SILVA, M. C. C.; MEDEIROS, J. F.; ESPÍNOLA SOBRINHO, J.; NEGREIROS, M. Z.. Estimativa da área foliar do meloeiro. Horticultura Brasileira, Brasília, v.20, n.4, p.555-558, 2002.

QUEIROGA, R. C. F.; PUIATTI, M.; FONTES, P. C. R.; CECON, P. R.. Produtividade e qualidade de frutos de meloeiro variando número de frutos e de folhas por planta. Horticultura Brasileira, Brasília, v.26, p.209-215, 2008.
REGAZZI, A. J.; LEITE, H. G.. Teste para verificar identidade de modelos de regressão. Pesquisa Agropecuária Brasileira, Brasília, v.31, n.1, p.1-17, 1996.

ROSELL, J.; SANZ, R.. A review of methods and applications of the geometric characterization of tree crops in agricultural activities. Computers and Electronics in Agriculture, v.81, p.124-141, 2012. DOI: http://doi.org/10.1016/j.compag.2011.09.007

SANZ, R.; LLORENS, J.; ESCOLA, A.; ARNO, J.; PLANAS, S.; ROMAN, C.; ROSELL-POLO, J. R.. LIDAR and non-LIDAR-based canopy parameters to estimate the leaf area in fruit trees and vineyard. Agricultural and Forest Meteorology, v.260261, p.229-239, 2018. DOI: http://doi.org/10.1016/j.agrformet.2018.06.017

SCHMILDT, E. R.; TREVISAN, E.; BELIQUE, M.; SCHMILDT, O. Modelos alométricos para determinação da área foliar de cacaueiro 'PH-16' em sombreamento e pleno sol. Revista Agroambiente, Roraima, v.11, n.1, p.47-55, 2017. DOI: http://doi.org/10.18227/1982-8470ragro.v11i1

SOUZA, A. L. P.; COSTA, M. M.; SENA JUNIOR, D. G.; PAZ, R. B. O.. Avaliação de três métodos de obtenção do índice de área foliar para cultura da soja. Nativa, Mato Grosso, v.7, n.3, p.284-287, 2019. DOI: http://dx.doi.org/10.31413/nativa.v7i3.7545

SPANN, T. M.; HEEREMA, R. J.. A simple method for nondestructive estimation of total shoot leaf area in tree fruit crops. Scientia Horticulturae, v.125, n.3, p.528-533, 2010. DOI: http://doi.org/10.1016/j.scienta.2010.04.033

WU, Z.; TU, M.; YANG, M.; XU, J.; YU, Z.. Effect of cutting and storage temperature on sucrose and organic acids metabolism in postharvest melon fruit. Postharvest Biology and Technology, v.161, p.1-12, 2020. DOI: http://doi.org/10.1016/j.postharvbio.2019.111081

ZEIST, A. R.; OLIVEIRA, J. R. F.; LIMA FILHO, R. B.; SILVA, M. L. S.; RESENDE, J. T. V.. Comparação de métodos de estimativa de área foliar em morangueiro. Pesquisa Agropecuária Gaúcha, Porto Alegre, v.20, n.1-2, p.33-40, 2014.

A CBPC - Companhia Brasileira de Produção Científica (CNPJ: 11.221.422/0001-03) detém os direitos materiais desta publicação. Os direitos referem-se à publicação do trabalho em qualquer parte do mundo, incluindo os direitos às renovações, expansões e disseminações da contribuição, bem como outros direitos subsidiários. Todos os trabalhos publicados eletronicamente poderão posteriormente ser publicados em coletâneas impressas sob coordenação da Sustenere Publishing, da Companhia Brasileira de Produção Científica e seus parceiros autorizados. Os (as) autores (as) preservam os direitos autorais, mas não têm permissão para a publicação da contribuição em outro meio, impresso ou digital, em português ou em tradução. 